\title{
FATORES PESSOAIS, DESPORTIVOS E PSICOLÓGICOS NO COMPORTAMENTO DE EXERCÍCIO FÍSICO
}

\author{
PERSONAL, ATHLETIC AND PSYCHOLOGICAL FACTORS IN EXERCISE BEHAVIOR \\ FACTORES PERSONALES, DEPORTIVOS Y PSICOLÓGICOS EN EL EJERCICIO FÍSICO
}

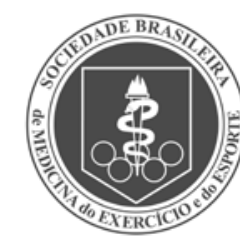

Artigo Original

ORIGINAL ARTICLE

Artículo Original
Luís Carneiro' (Psicólogo)

António Rui Gomes ${ }^{1}$ (Psicólogo)

1. Escola de Psicologia. Universidade do Minho, Braga, Portugal.

\section{Corespondência:}

Universidade do Minho Escola de Psicologia Rui Gomes - Campus de Gualtar. 4710-057. Braga. Portugal. rgomes@psi.uminho.pt

\section{RESUMO}

Introdução: os benefícios do exercício físico (EF) são bem conhecidos. Contudo, mais de $60 \%$ da população mundial é fisicamente inativa ou ativa de forma insuficiente para atingir tais vantagens. Nesse sentido, torna-se importante compreender os fatores envolvidos na prática de $\mathrm{EF}$, de modo a promover a participação desportiva e a prevenir o abandono do exercício. Objetivo: analisar as diferenças num conjunto de variáveis psicológicas em função da tendência para efetuar EF e as variáveis preditoras do comportamento efetivo de EF. Métodos: participaram no estudo 454 praticantes de musculação, cardiofitness e atividades de ritmo de uma academia desportiva no norte de Portugal, tendo sido avaliados três conjuntos de variáveis: a) pessoais (sexo, idade e índice de massa corporal); b) desportivas (tempo anterior de prática e frequência semanal de prática anterior ); e c) psicológicas (atitudes diante do EF, normas subjetivas diante do EF, percepção de controle comportamental, intenção de prática, benefícios e custos do EF e planejamento da prática). Resultados: os que praticam exercício com maior regularidade apresentaram níveis mais elevados de percepção de controle comportamental, intenção de prática e planejamento do EF, em comparação com os praticantes com atividade pouco ou nada regular. A idade, o tempo anterior de prática, a frequência semanal de prática anterior, as normas subjetivas e a percepção de controle comportamental foram preditores significativos do comportamento efetivo de EF. Conclusão: este estudo confirmou que os praticantes mais regulares evidenciaram um padrão psicológico mais favorável com relação à possibilidade de assumirem comportamentos estáveis de exercício. Por outro lado, os resultados demonstraram a natureza multifacetada dos fatores envolvidos no comportamento de EF.

Palavras-chave: exercício, academias de ginástica, comportamentos saudáveis, motivação.

\section{ABSTRACT}

Introduction: the benefits of exercise are well known; however more than $60 \%$ of the world population is physically inactive or insufficiently active to achieve such benefits. Therefore, it is important to understand the factors involved in exercise in order to promote participation and avoid discontinuation. Objective: to analyze the differences in a set of psychological variables according the tendency to do exercise and the predictors of actual exercise behavior. Methods: this study involved 454 practitioners of bodybuilding, cardio-fitness and rhythm activities of a sport center in the north of Portugal. Three sets of variables were evaluated: a) personal (sex, age, and body mass index); b) athletic (previous practice time and weekly frequency of previous practice); and c) psychological (attitudes toward exercise, subjective norms toward exercise, perceived behavioral control, exercise purpose, pros and cons of exercise, and exercise planning). Results: those who practice exercise more regularly had higher levels of perceived behavioral control, intention to practice, and exercise planning compared with practitioners with little or no exercise regular activity. Age, previous practice time, weekly frequency of previous practice, subjective norms and perceived behavioral control were significant predictors of the actual exercise behavior. Conclusion: this study confirmed that more regular practitioners showed a more favorable psychological pattern regarding the possibility of assuming stable exercise behavior. Moreover, the results demonstrated the multifaceted nature of the factors involved in exercise behavior.

Keywords: exercise, fitness centers, health behavior, motivation.

\section{RESUMEN}

Introducción: los beneficios del ejercicio físico (EF) son bien conocidos. Sin embargo, más del $60 \%$ de la población mundial es físicamente inactiva o insuficientemente activa para conseguir tales ventajas. En ese sentido, es importante entender los factores implicados en la práctica del EF, de manera a promover la participación en el deporte y prevenir el abandono del ejercicio. Objetivo: analizar las diferencias en un conjunto de variables psicológicas en función de la predisposición a realizar EF y los predictores del comportamiento efectivo de EF. Métodos: este estudio analizó 454 practicantes de musculación, cardiofitness y actividades de ritmo de una academia deportiva en el norte de Portugal. Se evaluaron tres conjuntos de variables: a) personales (sexo, edad e índice de masa corporal); b) deportivas (tiempo de la práctica anterior y frecuencia semanal de la 
práctica anterior) y c) psicológicas (actitudes hacia el EF, normas subjetivas hacia el EF, percepción del control de conducta, intención de práctica, costos y beneficios del EF y la planificación de la práctica. Resultados: los participantes con mayor regularidad de práctica de EF presentaron niveles más altos de percepción de control de conducta, intención de práctica y planificación de EF, en comparación con los participantes poco o nada regulares en la práctica. La edad, el tiempo de práctica anterior, la frecuencia semanal de la práctica anterior, las normas subjetivas y la percepción de control de conducta fueron predictores significativos del comportamiento efectivo de EF. Conclusión: este estudio confirmó que más participantes regulares mostraron un patrón psicológico más favorable en cuanto a la a la posibilidad de asumir un comportamiento estable de ejercicio. Además, los resultados demostraron la naturaleza polifacética de los factores implicados en el comportamiento del EF.

Palabras clave: ejercicio, centros de acondicionamiento, conductas saludables, motivación.

\section{INTRODUÇÃO}

Os benefícios do exercício físico são bem conhecidos ${ }^{1,2}$. Todavia, segundo a WHO Global Strategy on Diet, Physical Activity and Health, mais de $60 \%$ da população mundial é fisicamente inativa ou ativa de forma insuficiente para a obtenção de tais benefícios ${ }^{3}$. Além disso, é preocupante o facto de, em média, 50\% dos indivíduos que iniciam um programa de exercício físico regular desistirem nos primeiros seis meses ${ }^{4}$. Neste sentido, torna-se relevante compreender melhor quais os fatores que melhor diferenciam os indivíduos que se mantêm na prática de exercício físico daqueles que infelizmente abandonam este comportamento. Aliás, como tem vindo a ser salientado na literatura são menos os estudos que abordam a manutenção deste comportamento ${ }^{4}$ relativamente àqueles que se centram nos fatores explicativos do início da prática de exercício físico ${ }^{5}$. Assim sendo, o primeiro objetivo deste estudo foi analisar os aspetos psicológicos que tendem a diferenciar as pessoas que se mantêm e abandonam a prática de exercício, procurando-se assim contribuir para um melhor entendimento dos fatores implicados na manutenção de estilos de vida saudável.

Um outro problema existente no estudo do comportamento de exercício físico, prende-se com a importância de analisar não apenas os fatores que condicionam a intenção de praticar exercício, mas antes tentar compreender os fatores envolvidos na explicação do comportamento efetivo de exercício físico ${ }^{5}$. Esta necessidade decorre do facto de não existir uma relação evidente entre a afirmação e/ou previsão das pessoas acerca do assumir um dado comportamento de exercício e o subsequente comportamento efetivo de exercício. Dito por outras palavras, a conversão das intenções no comportamento objetivo de exercício não se encontra bem determinado pelos modelos psicológicos atuais ${ }^{6}$, sendo que as variáveis psicológicas têm demonstrado uma maior capacidade para explicar a intenção de prática de exercício do que propriamente o comportamento de exercício físico ${ }^{7-9}$. Deste modo, existe uma falta de relação entre a intenção e o comportamento de exercício, sendo este fenómeno descrito na literatura como "intention-behavior gap", representando um dos maiores desafios que se coloca à investigação no domínio dos fatores psicológicos associados ao exercício físico ${ }^{6}$. Foi tendo por base este problema que organizamos o segundo objetivo deste trabalho, centrando as análises realizadas de acordo com o comportamento efetivo de exercício físico dos participantes neste estudo, procurando estudar os fatores que predizem este hábito de vida saudável. Para tal, recolhemos dados acerca do comportamento efetivo de exercício físico assumido pelos participantes, utilizando-se para este efeito o registo eletrónico de exercício numa academia desportiva.
Na tentativa de respondermos de um modo o mais abrangente possível a estas duas questões, incluímos nas análises três tipos de variáveis. Em primeiro lugar, observamos dimensões de caráter pessoal, nomeadamente o sexo, a idade e o índice de massa corporal (IMC). Estas características dos participantes foram selecionadas devido ao seu papel central na investigação neste domínio ${ }^{10-13}$, procurando-se neste trabalho testar o seu papel preditivo no comportamento de exercício físico, respondendo assim ao segundo objetivo deste trabalho.

Em segundo lugar, incluímos variáveis de natureza desportiva, de que são exemplo o tempo anterior de prática de exercício físico e a frequência semanal anterior de prática de exercício físico. A este nível, existem indicações sobre o papel relevante do comportamento (ou tempo) anterior de exercício físico na explicação da manutenção

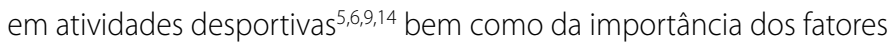
desportivos (ou atléticos) na frequência semanal de exercício físico ${ }^{10}$. Tal como nas variáveis pessoais, estas dimensões foram incluídas neste trabalho de modo a responder ao segundo objetivo formulado.

Em terceiro lugar, incluímos facetas de natureza psicológica para responder a ambos os objetivos deste estudo, selecionando para o efeito dimensões representativas de três modelos conceptuais bem conhecidos na explicação de comportamentos de saúde em geral e do comportamento de exercício físico em particular. Mais concretamente, foram incluídas as variáveis centrais da Teoria do Comportamento Planeado (TCP) ${ }^{15}$ (e.g., atitudes, normas subjetivas, perceção de controle comportamental e intenção). A este nível, a TCP é considerada uma das propostas teóricas mais compreensivas da explicação da alteração dos hábitos de saúde, como é o caso do comportamento de exercício físico ${ }^{16}$. De um modo geral, o modelo defende que a intenção para assumir um determinado comportamento e a perceção de controle comportamental são preditores diretos do comportamento em causa e que a intenção comportamental é, por sua vez, influenciada pelas atitudes, normas subjetivas e perceção de controle comportamental ${ }^{17}$.

A segunda proposta conceptual considerada foi o modelo transteórico das etapas de mudança, incluindo-se neste estudo um dos seus conceitos fundamentais relacionado com as vantagens (prós) e das desvantagens (contras) da mudança de comportamento. Este conceito representa uma das condições necessárias para que a mudança comportamental seja efetuada com sucesso ${ }^{18}$, tendo vindo a ganhar sustentação em contextos de exercício físico ${ }^{19}$.

A terceira, e última variável estudada, foi o planeamento da prática de exercício, que é uma dimensão integrante do modelo de ação na saúde (Health Action Process Approach - HAPA) ${ }^{20}$. Uma vez mais, esta variável tem vindo a ser estudada ao nível do exercício físico, dando indicações favoráveis na sua capacidade para explicar este comportamento ${ }^{21}$. 
Em suma, e considerando todos estes aspetos, este estudo teve dois objetivos específicos: a) Analisar as diferenças nas variáveis psicológicas em função do comportamento efetivo de exercício físico assumido pelos participantes; e b) Analisar as variáveis preditoras do comportamento efetivo de exercício físico.

\section{MÉTODOS}

Participaram neste trabalho 454 praticantes de exercício físico, das modalidades de musculação, cardio-fitness e atividades de ritmo, de uma academia desportiva do norte de Portugal. A maioria dos participantes era do sexo feminino $(n=248,54.7 \%)$, sendo $45.3 \%$ dos participantes do sexo masculino $(n=205)$. Quanto à idade, esta variou entre os 15 e os 61 anos $(M=25.14 ; D P=7.13)$. Em termos do tempo anterior de prática de exercício físico, 120 participantes efetuavam exercício num período que variou entre um a seis meses (26.5\%), 54 efetuavam exercício há mais de seis meses até um ano (11.9\%), 86 efetuavam exercício há mais de um ano até cinco anos (19\%) e 193 efetuavam exercício há mais de cinco anos (42.6\%). No que se refere à frequência semanal anterior de prática de exercício, oito dos participantes não realizavam exercício físico (1.8\%), 216 realizavam exercício até duas vezes por semana (47.8\%), 207 realizavam exercício até cinco vezes por semana (45.8\%) e 21 realizavam exercício cinco ou mais vezes por semana (4.6\%).

Informação demográfica: este instrumento foi desenvolvido para este estudo, avaliando variáveis pessoais (e.g., sexo, idade, peso e altura atuais) e desportivas (e.g., tempo anterior de exercício físico e frequência semanal anterior de exercício físico).

Atitudes face ao exercício (AFE) ${ }^{7}$ : Este instrumento baseou-se na proposta de Ajzen ${ }^{22}$, avaliando as dimensões instrumental (ex: "Útil//nútil") e afetiva (ex: "Agradável/Desagradável") da prática de exercício físico. Para tal, foram colocados seis pares de adjetivos que refletem ambas as dimensões, sendo apresentados em formato de escalas avaliativas de diferencial semântico (e.g., bipolares com sete pontos).

Normas subjetivas face ao exercício físico (NSEF)23: este instrumento baseou-se na proposta de Ajzen ${ }^{22}$, avaliando as crenças normativas da pessoa face à opinião geral que os outros têm relativamente à sua prática de exercício físico (ex: "A maioria das pessoas que são importantes para mim apoia a minha prática regular de exercício físico"). Para tal, foram apresentados quatro itens respondidos numa escala tipo "Likert" de sete pontos ( $1=$ Discordo totalmente; $7=$ Concordo totalmente).

Perceção de controle comportamental $(P C C)^{7}$ : este instrumento baseou-se na proposta de Ajzen ${ }^{22}$, representado uma medida global da perceção de controle comportamental que a pessoa assume face à prática efetiva de exercício físico ${ }^{24}$. Para tal, foram apresentados quatro itens que avaliam o comportamento de exercício nos próximos três meses com uma frequência de prática de, pelo menos, três vezes por semana (ex: "Fazer exercício físico, pelo menos três vezes por semana, será algo de..."). As respostas foram dadas numa escala tipo "Likert" de sete pontos (ex: 1=Muito difícil; 7=Muito fáci).

Intenção de prática de exercício físico (IPEF) 23: este instrumento baseou-se na proposta de Ajzen ${ }^{22}$, avaliando a intenção de prática de exercício físico. Esta medida avaliou a ação propriamente dita (e.g., "fazer exercício físico"), o contexto concreto de prática (e.g., "ginásio") e o tempo específico de prática de exercício (e.g., "nos próximos três meses"). Para tal, foram apresentados três itens numa escala tipo "Likert" de sete pontos ( $1=$ Nenhuma certeza; $7=$ Certeza absoluta).

Benefícios e custos do exercício físico (BCEF) ${ }^{23}$ : o instrumento teve por base os trabalhos desenvolvidos sobre os fatores psicológicos associados ao exercício físico ${ }^{19}$, avaliando até que ponto a prática de exercício físico é percecionada pelo indiví- duo como assumindo um conjunto de consequências positivas (benefícios ou prós do exercício) e, inversamente, de consequências negativas (custos ou contras do exercício). Para tal, foram apresentados oito itens respondidos numa escala tipo "Likert" de sete pontos ( $1=$ Discordo totalmente; $7=$ Concordo totalmente). Os quatro primeiros itens são relativos aos benefícios/prós do exercício (ex: "Sentir que fiz algo de positivo pela minha saúde") e os quatro últimos itens referem-se aos custos/contras do exercício (ex: "Não conseguir fazer todas as minhas tarefas habituais").

Planeamento da prática de exercício físico (PPEF) ${ }^{23}$ : este instrumento teve por base trabalhos de Norman e Conner ${ }^{9}$ e de Lippke et al. ${ }^{25}$, avaliando o nível de planeamento assumido pela pessoa relativamente à prática de exercício. Mais concretamente, o instrumento avalia o momento, o contexto, o modo e a frequência concreta de exercício físico (ex: "Tenho bem definido onde farei exercício físico"). Para tal, foram apresentados quatro itens respondidos numa escala tipo "Likert" de sete pontos (1=Discordo totalmente; 7=Concordo totalmente).

Este estudo foi realizado de acordo com as normas de investigação com seres humanos defendidas pelo Centro de Investigação em Psicologia da Universidade do Minho, Portugal, sendo que estas normas estão de acordo com os regulamentos nacionais e europeus que regem a investigação com seres humanos e a gestão de dados pessoais. O trabalho iniciou-se com o contacto do responsável pela academia desportiva, explicando-se os objetivos do estudo e o procedimento de recolha de dados. Após a obtenção da autorização para a realização do estudo, efetuámos a recolha de dados junto dos participantes. O protocolo de avaliação incluiu uma carta explicativa dos objetivos do estudo, assegurando-se o caráter voluntário da participação e a confidencialidade do tratamento dos dados. Após esta informação, foi obtido o consentimento informado. A recolha de dados decorreu em duas fases. Assim, nos meses de Outubro a Dezembro de 2011 foi aplicado o protocolo de avaliação com os instrumentos descritos. Repare-se que os participantes responderam ao protocolo de avaliação pensando exclusivamente neste contexto de prática de exercício físico e para um período médio de três meses. Após terem decorrido estes três meses, efetuamos uma análise da frequência de prática de exercício físico relativas aos meses de Janeiro, Fevereiro e Março de 2012, recorrendo-se aos registos informáticos de entrada dos praticantes na academia desportiva. Deste modo, obteve-se uma relação entre as medidas de autorrelato do protocolo de avaliação e as medidas efetivas de exercício físico. Em termos de participação no estudo, foram entregues 651 protocolos, tendo sido devolvidos 454, obtendo-se uma taxa de retorno de $69.7 \%$.

\section{RESULTADOS}

Antes de se proceder às análises centrais deste estudo, observámos a estrutura fatorial de todos os instrumentos utilizados. Para tal, efetuou-se uma análise fatorial das componentes principais, sem predefinição do número de fatores, através da rotação ortogonal (procedimento "varimax"), com normalização de Kaiser ("eigenvalue" igual ou superior a 1). De seguida, observámos a consistência interna de cada um dos fatores observados através dos valores de "alpha" de Cronbach. Os valores obtidos do KMO ("Kaiser-Meyer-Olkin") e do teste de esfericidade de Bartlett indicaram a adequabilidade da aplicação deste procedimento de análise a todos os instrumentos, tendo estes revelado as estruturas fatoriais previstas. De igual modo, os valores de fidelidade ("alpha" de Cronbach) assumiram valores bastante aceitáveis nas dimensões dos instrumentos (valores acima de 0.70). 


\section{Diferenças nas variáveis psicológicas em função do comportamen- to efetivo de exercício}

Esta análise diz respeito ao primeiro objetivo do estudo, procurando-se observar se existiam diferenças nas dimensões psicológicas avaliadas no protocolo de avaliação (e.g., atitudes, normas subjetivas, perceção de controle comportamental, intenção de prática de exercício, prós e contras do exercício, planeamento da prática de exercício físico) em função da frequência de exercício físico assumido pelos participantes. Para a criação de grupos de comparação em função da frequência de exercício físico, começamos por analisar os valores médios de exercício nos três meses em causa. Em termos médios, a frequência variou entre 0 e 25 vezes por mês ( $M=4.75 ; D P=4.65)$. Neste sentido, foram constituídos três grupos de comparação: (a) praticantes sem frequência de exercício físico $(n=71,15.6 \%)$; (b) praticantes com menor regularidade de exercício físico (e.g., até 2 vezes por semana; $n=285,62.8 \%)$ ); e (c) praticantes com maior regularidade de exercício físico (3 ou mais vezes por semana; $n=98,21.6 \%$ ). A constituição dos grupos foi realizada com base nas recomendações sugeridas pelo American College of Sports Medicine ${ }^{26}$.

As análises comparativas foram efetuadas através de testes de diferenças inter-sujeitos (One-way ANOVA) e de análises multivariadas da variância (MANOVA), tendo-se confirmado os pressupostos da normalidade da distribuição dos resultados antes de realizar estas comparações. Os resultados obtidos com os testes referidos indicaram diferenças com significado estatístico ao nível da perceção de controle comportamental, da intenção de prática de exercício físico e do planeamento da prática de exercício. Os testes "post-hoc" de Scheffe revelaram que os participantes com maior regularidade de exercício físico assumiram uma maior perceção de controle comportamental, uma maior intenção de prática de exercício e um maior planeamento da prática de exercício físico (tabela 1).

Tabela 1. Diferenças nas variáveis psicológicas em função da prática de exercício físico

\begin{tabular}{|c|c|c|c|c|c|}
\hline Variável & $\begin{array}{c}\text { Sem } \\
\text { regularidade } \\
M(D P)(n)\end{array}$ & $\begin{array}{c}\text { Menor } \\
\text { regularidade } \\
M(D P)(n)\end{array}$ & $\begin{array}{c}\text { Maior } \\
\text { regularidade } \\
M(D P)(n)\end{array}$ & g.l & $F$ \\
\hline $\begin{array}{c}\text { AFE: } \\
\text { Instrumental }\end{array}$ & $6,73(0,47)(70)$ & $6,75(0,47)(275)$ & $6,71(0,86)(94)$ & 2,436 & 0,22 n.s. \\
\hline AFE: Afetiva & $5,99(1,18)(70)$ & $6,12(0,93)(275)$ & $6,31(1,04)(94)$ & 2,436 & 2,11 n.s. \\
\hline $\begin{array}{l}\text { NSEF: Normas } \\
\text { subjetivas }\end{array}$ & $5,29(1,17)(71)$ & $5,49(1,25)(282)$ & $5,18(1,38)(98)$ & 2,448 & 2,41 n.S. \\
\hline $\begin{array}{c}\text { PCC: Perceção } \\
\text { controle } \\
\text { comportamental }\end{array}$ & $4,64(1,50)(71)$ & $4,94(1,36)(285)$ & $5,67(1,13)(98)$ & 2,451 & $14,88^{* * *}$ \\
\hline IPEF: Intenção & $4,50(1,74)(71)$ & $4,79(1,73)(285)$ & $5,68(1,31)(98)$ & 2,451 & $13,67^{* * *}$ \\
\hline BCEF: Prós & $5,86(0,82)(71)$ & $5,97(0,77)(284)$ & $6,08(0,84)(98)$ & 2,450 & 1,69 n.s. \\
\hline BCEF: Contras & $2,50(1,22)(71)$ & $2,37(1,19)(284)$ & $2,43(1,27)(98)$ & 2,450 & 0,33 n.s. \\
\hline $\begin{array}{c}\text { PPEF: } \\
\text { Planeamento }\end{array}$ & $5,38(1,29)(71)$ & $5,47(1,17)(284)$ & $5,91(0,99)(98)$ & 2,450 & $6,17^{* *}$ \\
\hline
\end{tabular}

\section{Predição do comportamento efetivo de exercício físico}

O objetivo desta análise foi testar o valor preditivo dos três conjuntos de variáveis definidas para este estudo (e.g., pessoais, desportivas e psicológicas) na explicação do comportamento efetivo de exercício físico, constituindo-se para o efeito um valor final relativo aos três meses de avaliação. Assim sendo, começámos por analisar separadamente cada um dos três conjuntos de variáveis (modelos de regressão inicial) e, num último modelo de regressão (modelo de regressão final), incluímos apenas as variáveis que tinham assumido um valor preditivo nos modelos iniciais na explicação do comportamento de exercício físico. Para este efeito, realizámos análises de regressão hierárquica (método "enter").

Começando pelos modelos de regressão inicial (tabela 2), nas variáveis pessoais o sexo e a idade foram preditores do comportamento efetivo de exercício físico, explicando $6 \%$ da variância final. Neste caso, o modelo foi obtido após a retirada de seis outliers. Nas variáveis desportivas, ambas as dimensões foram preditoras do comportamento efetivo de exercício físico, explicando 17\% da variância final. Neste caso, o modelo foi obtido após a retirada de quatro outliers. Nas variáveis psicológicas, as normas subjetivas e a perceção de controle comportamental foram preditoras do comportamento efetivo de exercício físico, explicando $12 \%$ da variância final. Neste caso, o modelo foi obtido após a retirada de quatro outliers.

Tabela 2. Modelos de regressão inicial para a predição do comportamento efetivo de exercício físico.

\begin{tabular}{|c|c|c|c|c|}
\hline & $R^{2}$ ( $R^{2}$ ajust.) & $F$ & $\beta$ & $t$ \\
\hline Modelo 1: Variáveis pessoais & $0,07(0,06)$ & $\begin{array}{l}(3,409) \\
9,75^{* * *}\end{array}$ & & \\
\hline Sexo (a) & & & $-0,15$ & $-2,99 * *$ \\
\hline Idade & & & 0,21 & $4,31^{* * *}$ \\
\hline IMC (b) & & & 0,00 & 0,02 n.s \\
\hline Modelo 2: Variáveis desportivas & $0,17(0,17)$ & $\begin{array}{c}(2,444) \\
44,94^{* * *}\end{array}$ & & \\
\hline Tempo anterior de prática de EF & & & 0,18 & $4,20^{* * *}$ \\
\hline Frequência semanal anterior de EF & & & 0,34 & $7,66^{* * *}$ \\
\hline Modelo 3: Variáveis psicológicas & $0,13(0,12)$ & $\begin{array}{c}(5,426) \\
12,97^{* * *}\end{array}$ & & \\
\hline \multicolumn{5}{|l|}{ Bloco 1} \\
\hline AFE: Instrumental & & & $-0,10$ & $-1,77$ n.s. \\
\hline AFE: Afetiva & & & 0,09 & 1,61 n.s. \\
\hline NSEF: Normas subjetivas & & & $-0,16$ & $-3,54^{* * *}$ \\
\hline PCC: Perceção controle comportamental & & & 0,18 & $2,22^{*}$ \\
\hline IPEF: Intenção & & & 0,13 & 1,60 n.s. \\
\hline Bloco 2 & $0,14(0,12)$ & $\begin{array}{l}(7,424) \\
9,46^{* * *}\end{array}$ & & \\
\hline AFE: Instrumental & & & $-0,09$ & $-1,68$ n.s \\
\hline AFE: Afetiva & & & 0,08 & 1,35 n.s. \\
\hline NSEF: Normas subjetivas & & & $-0,17$ & $-3,55^{* * *}$ \\
\hline PCC: Perceção controle comportamental & & & 0,18 & $2,13^{*}$ \\
\hline IPEF: Intenção & & & 0,13 & 1,60 n.s. \\
\hline BCEF: Prós & & & 0,05 & 0,91 n.s. \\
\hline BCEF: Contras & & & 0,04 & 0,90 n.s. \\
\hline Bloco 3 & $0,14(0,12)$ & $\begin{array}{l}(8,423) \\
8,34^{* * *} \\
\end{array}$ & & \\
\hline AFE: Instrumental & & & $-0,09$ & $-1,70+$ \\
\hline AFE: Afetiva & & & 0,08 & 1,36 n.s. \\
\hline NSEF: Normas subjetivas & & & $-0,17$ & $-3,59 * * *$ \\
\hline PCC: Perceção controle comportamental & & & 0,17 & $1,92^{*}$ \\
\hline IPEF: Intenção & & & 0,13 & 1,54 n.s. \\
\hline BCEF: Prós & & & 0,04 & 0,86 n.s. \\
\hline BCEF: Contras & & & 0,04 & 0,83 n.s. \\
\hline PPEF: Planeamento & & & 0,04 & 0,74 n.s. \\
\hline
\end{tabular}

(a)Sexo:0-Masculino,1-Feminino; ${ }^{\text {(b) }}$ IMC: 0-Peso normal, 1-Excesso de peso. ${ }^{*} p<.05 ;{ }^{* *} p<.01 ;{ }^{* * *} p<.001$. 
De seguida, e verificadas as variáveis dos três modelos anteriores que se mostraram significativas na predição do comportamento efetivo de exercício físico, foi testado um modelo de regressão final, considerando-se agora três blocos de entrada com as variáveis pessoais, desportivas e psicológicas (tabela 3). Começando pelo bloco um, tanto o sexo como a idade foram preditores do comportamento efetivo de exercício físico, explicando 7\% da variância final. Assim, ser do sexo masculino e ter maior idade foi preditor de uma maior frequência de prática efetiva de exercício físico. No bloco dois, ambas as dimensões foram preditoras do comportamento efetivo de exercício físico, explicando o modelo 19\% da variância final. Neste caso, ter maior tempo anterior de prática de exercício físico e ter uma maior frequência semanal de sessões de exercício físico foram preditores do comportamento efetivo de exercício físico. Finalmente, no bloco três acrescentaram-se as dimensões psicológicas, passando o modelo a explicar 23\% da variância final. Mais concretamente, a menor pressão social percebida para fazer exercício (e.g., normas subjetivas) e a maior perceção de controle comportamental foram preditores da frequência de prática efetiva de exercício físico. Convém referir que neste bloco final a variável sexo perdeu o seu valor preditivo. Este modelo final foi obtido após a retirada de sete outliers.

Tabela 3. Modelos de regressão final para a predição do comportamento efetivo de exercício físico.

\begin{tabular}{|c|c|c|c|c|}
\hline & $R^{2}$ ( $R^{2}$ ajust.) & $F$ & $\beta$ & $t$ \\
\hline Bloco 1: Variáveis pessoais & $0,07(0,07)$ & $\begin{array}{c}(2,426) \\
15,99^{* * *}\end{array}$ & & \\
\hline Sexo & & & $-0,14$ & $-2,89^{* *}$ \\
\hline Idade & & & 0,23 & $4,88^{* * *}$ \\
\hline Bloco 2: Variáveis desportivas & $0,20(0,19)$ & $\begin{array}{c}(4,424) \\
26,39^{* * *}\end{array}$ & & \\
\hline Sexo & & & $-0,05$ & $-1,13$ n.S. \\
\hline Idade & & & 0,20 & $4,64^{* * *}$ \\
\hline Tempo anterior de prática de EF & & & 0,19 & $4,09^{* * *}$ \\
\hline Frequência semanal anterior de EF & & & 0,29 & $6,50^{* * *}$ \\
\hline Bloco 3: Variáveis psicológicas & $0,24(0,23)$ & $\begin{array}{c}(6,422) \\
22,24^{* * *}\end{array}$ & & \\
\hline Sexo & & & $-0,03$ & $-0,68$ n.s. \\
\hline Idade & & & 0,21 & $4,93^{* * *}$ \\
\hline Tempo anterior de prática de EF & & & 0,17 & $3,70^{* * *}$ \\
\hline Frequência semanal anterior de EF & & & 0,20 & $3,81^{* * *}$ \\
\hline NSEF: Normas Subjetivas & & & $-0,16$ & $-3,73^{* * *}$ \\
\hline PCC: Perceção contro. comporta. & & & 0,17 & $3,35^{* *}$ \\
\hline
\end{tabular}

\section{DISCUSSÃO}

Este estudo analisou, por um lado, a existência de diferenças do ponto de vista psicológico em função do comportamento efetivo de exercício físico assumido pelos participantes e, por outro lado, analisou as variáveis preditoras do comportamento efetivo de exercício físico.

Começando pelo primeiro objetivo, verificou-se que os praticantes com maior regularidade na prática de exercício físico evidenciaram uma maior perceção de controle comportamental face à possibilidade de fazerem exercício, uma maior intenção em assumir este comportamento e um maior planeamento desta atividade relativamente aos praticantes que entretanto desistiram desta atividade e aos que assumiram uma menor frequência de exercício físico. Repare-se que estes dados são tão mais relevantes pois foram obtidos num momento em que todos os grupos de comparação efetuavam exercício físico, vindo estas diferenças a assumir-se como diferenciadoras entre os participantes após três meses de atividade desportiva. Relativamente à perceção de controle comportamental e à intenção do exercício físico, é interessante realçar que ambas as dimensões ocupam um papel central no modelo da Teoria do Comportamento Planeado ${ }^{15}$, sendo ambas determinantes fundamentais do comportamento futuro das pessoas. Quanto à diferença encontrada no planeamento da prática de exercício, existem também indicações da literatura que sugerem que esta variável pode efetivamente diferenciar indivíduos que se encontram na fase não intencional da prática de exercício relativamente aos que se encontram nas fases intencional e de ação de exercício ${ }^{25}$.

Relativamente ao segundo objetivo, no modelo final de regressão verificou-se que a frequência efetiva de exercício físico foi predita pelo sexo (embora no bloco três tenha deixado de ser significativo), idade, tempo anterior de prática de exercício físico, frequência semanal anterior de exercício físico, normas subjetivas e perceção de controle comportamental. Algumas destas variáveis têm vindo a ser evidenciadas na literatura como importantes para a explicação da prática de exercício. No caso das diferenças em função do sexo, os dados deste estudo estão de acordo com indicações da literatura que sugerem a tendência das mulheres para assumirem menores padrões de exercício físico ${ }^{13}$. No caso da idade, dados de meta-análises sugerem que o avançar da idade das pessoas aumenta a probabilidade destas converterem as suas intenções no comportamento efetivo de exercício físico ${ }^{8}$. Quanto às variáveis desportivas deste estudo, também existem indicações que o comportamento (ou tempo) anterior de exercício tem sido referenciado como um dos melhores preditores do comportamento futuro ${ }^{8}$. Já nas variáveis psicológicas, um dos dados mais surpreendentes deste estudo prendeu-se com o facto das normas subjetivas terem assumido um valor preditivo significativo, uma vez que esta variável tem sido apontada na literatura como um preditor fraco da intenção e do comportamento de exercício ${ }^{8,18}$. É ainda curioso verificar que no nosso caso, ao contrário do que seria expectável e proposto pela Teoria do Comportamento Planeado, foi a menor pressão social percebida para fazer exercício que foi preditora de uma maior frequência de exercício físico. Não possuímos dados que nos permitam avançar com explicações categóricas para este resultado, mas pode-se levantar como hipótese a possibilidade dos participantes neste estudo assumirem uma vontade mais intrínseca em praticar exercício, recusando assim a influência externa dos outros para esta atividade. Outra possibilidade a não descurar será a possibilidade de fenómenos de desejabilidade social terem interferido na resposta a este instrumento. Finalmente, a perceção de controle comportamental também foi um preditor significativo do comportamento de exercício físico, existindo igualmente dados da investigação que apontam o papel importante desta variável na explicação do assumir estilos de vida ativos ${ }^{5,9}$.

\section{CONCLUSÕES}

Este estudo permitiu constatar que os praticantes regulares de exercício físico assumiram um padrão psicológico mais favorável relativamente à possibilidade de assumirem comportamentos estáveis de exercício. Paralelamente, também se verificou a importância das variáveis pessoais, desportivas e psicológicas na explicação do comportamento de exercício físico. 
Estes resultados deixam pistas para os estudos futuros que se queiram dedicar a um dos maiores dilemas da investigação neste domínio, que se prende com a compreensão da falta de relação entre a intenção de fazer exercício e o comportamento efetivo de exercício (intention-behavior gap). Os dados obtidos, apesar de avançarem na compreensão deste fenómeno, também deixam claro dois aspetos limitadores. Por um lado, nem todas as variáveis foram estatisticamente significativas nas análises realizadas (colocando-se assim em causa a sua relevância na compreensão do comportamento de exercício) e o modelo final de regressão acabou por não atingir níveis de variância explicada muito elevados (23\%). Seja como for, só será possível avançar neste domínio de estudo se analisarmos o comportamento efetivo de exercício e se assumirmos uma orientação multifacetada das variáveis estudadas, tal como foi proposto neste trabalho.

\section{AGRADECIMENTOS}

Os autores agradecem aos Serviços de Ação Social da Universidade do Minho, Braga, Portugal pelo apoio dado na realização deste trabalho.

Todos os autores declararam não haver qualquer potencial conflito de interesses referente a este artigo.

\section{REFERÊNCIAS}

1. Hardman AE, Stensel DJ. Physical activity and health: the evidence explained. 2nd ed. New York: Routledge; 2009.

2. Ruiz YP, Cañaveras DP. Actividad física y salud. In: Mendo AH. Psicología del deporte: aplicaciones 1. Buenos Aires: Efdeportes; 2003. p. 167-88.

3. World Health Organization. Physical inactivity: a global public health problem. 2008. Disponivel em: www.who.int/dietphysicalactivity/factsheet_inactivity/en/index.html. [Acesso em: 2012, Fevereiro, 12].

4. Marcus BH, Dubbert PM, Forsyth LH, McKenzie TL, Stone EJ, Dunn AL, et al. Physical activity behavior change: issues in adoption and maintenance. Health Psychol. 2000;19 (Suppl 1):32-41.

5. Armitage CJ. Can the theory of planned behavior predict the maintenance of physical activity? Health Psychol. 2005;24(3):235-45.

6. Mohiyeddini C, Pauli R, Bauer S. The role of emotion in bridging the intention-behaviour gap: the case of sports participation. Psychol Sport Exerc. 2009;10:226-34.

7. Gomes AR, Capelão T. Commitment to exercise: the influence of personal, athletic, and psychological characteristics. Univ Psychol. 2013;12(2):505-15.

8. Hagger MS, Chatzisarantis NLD, Biddle SJH. A meta-analytic review of the theories of reasoned action and planned behavior in physical activity: predictive validity and the contribution of additional variables. Psychol Sport Exerc. 2002;24:3-32.

9. Norman $\mathrm{P}$, Conner $\mathrm{M}$. The theory of planned behavior and exercise: evidence for the mediating and moderating roles of planning on intention-behavior relationships. J Sport Exerc Psychol. 2005;27:488-504.

10. Gomes AR, Capelão T. Predicting exercise behavior: testing personal, athletic, and psychological variables. Int J Sports Sci. 2012;2(5):45:50.

11. Janssen I, Katzmarzyk PT, Boyce WF, King MA, PickettW. Overweight and obesity in canadian adolescents and their associations with dietary habits and physical activity patterns. J Adolesc Health 2004;35(5):360-67.

12. Rhodes RE, Blanchard CM, Blacklock RE. Do physical activity beliefs differ by age and gender? I Sport Exerc Psychol. 2008;30(3):412-23.

13. Trost SG, Owen N, Bauman AE, Sallis JF, Brown W. Correlates of adults' participation in physical activity: review and update. Med Sci Sports Exerc 2002;34(12):1996-2001.
14. Hamilton $\mathrm{K}$, White KM. Extending the theory of planned behavior: the role of self and social influences in predicting adolescent regular moderate-to-vigorous physical activity. J Sport Exerc Psychol. 2008;30:56-74.

15. Ajzen I. The theory of planned behavior. Organ Behav Hum Decis Process. 1991;50(2):179-211.

16. Courneya KS, Bobick TM. Integrating the theory of planned behavior with the processes and stages of change in the exercise domain. J Sport Exerc Psychol. 2000;1:41-56.

17. Ajzen I. Attitudes, personality and behavior. 2nd ed. England: Open University Press; 2005.

18. Biddle SJ, Mutrie N. Psychology of physical activity: determinants, well-being \& interventions. 2nd. ed. London: Routledge; 2008.

19. Jordan PJ, Nigg CR, Norman GJ, Rossi JS, Benisovich SV. Does the transtheoretical model need an attitude adjustment? Integrating attitude with decisional balance as predictors of stage of change for exercise. Psychol Sport Exerc. 2002;3:65-83.

20. Biddle SJH, Hagger MS, Chatzisarantis NLD, Lippke S. Theoretical frameworks in exercise psychology. In: Tenenbaum G, Eklund RC, editors. Handbook of sport psychology. 3rd ed. Hoboken: John Wiley and Sons. 2007. p. 537-59.

21. Milne S, Orbell S, Sheeran P. Combining motivational and volitional interventions to promote exercise participation: protection motivation theory and implementation intentions. British J Health Psychol. 2002;7(2):163-84.

22. Ajzen I. Construction of a standard questionnaire for the theory of planned behaviour. 2002. Disponivel em: http://www-unix.oit.umass.edu/ aizen/. [Acesso em: 2011, Setembro, 12].

23. Gomes AR. Instrumentos de avaliação dos fatores associados ao exercício físico. [relatório técnico não publicado]. Braga: Universidade do Minho; 2011

24. Rhodes RE, Courneya KS, Hayduk LA. Does personality moderate the theory of planned bahavior in the exercise domain? J Sport Exerc Psychol. 2002;24:120-32.

25. Lippke S, Ziegelmann JP, Schwarzer R. Stage-specific adoption and maintenance of physical activity: testing a three-stage model. Psychol Sport Exerc. 2005;6:585-603.

26. American College of Sports Medicine. ACSM's guidelines for exercise testing and prescription. 8th ed. Baltimore: Lippincott Williams \& Wilkins; 2009. 